\title{
XXI.
}

Aus der psychiatrischen und Nervenklinik der Königl. Charité (Prof. Jolly).

\section{Ueber Veränderungen der Hirnrinde unter einem subduralen Hämatom.}

\author{
Von \\ Dr. M. Köppen, \\ a. o. Professor, erster Assistent der psychiatrischen Kilinik.
}

(Hierzu Tafel XVII.)

Im nachfolgenden möchte ich im Anschluss an die vorherstehende Arbeit die Beschreibung eines Falles geben, bei dem es sich ebenfalls um die Folge einer Schädelverletzung handelt. Allein das Interesse des Falles liegt in einer anderen Richtung als bei den bisher veröffentlichten.

Unmittelbar an der Stelle der Gewalteinwirkung war es zur Bildung eines Hämatoms gekommen und unter diesem Hämatom, durch das die ganze rechte Hemisphäre eingedrückt war, fanden sich weitaus verbreitete Veränderungen der Hirnrinde, die eine ausführliche Beschreibung verdienen.

Paul Gluske, 31 Jahre alt, Eisenbahnarbeiter. Aufgenommen am 9. Februar 1898, gestorbon am 8 . März 1898 .

GL. wurde am 5. Februar 1898 dadurch verletzt, dass ihm eine Kiste auf den Fuss fiel, die eine Quetschung der linken grossen Zehe herbeifübrte. Am 7. Februar 1898 fiel Gl. auf dem Bahnhof plötzlich um und schlug mit der Stirn auf den Rand einer Kiste auf. Aus der Wunde am Kopf und ans der Nase floss Blut. Wegen Ausbruch eines Deliriums musste er dann am 9. Febr. der Königl. Charité überwiesen werden.

Gl. befand sich bei der Einlieferung in die Charité in dem Zustand eines vollkommenen Deliriums. Er war unorientirt und hatte massenhafte Hallucinationen. Es bestand allgemeiner Tremor, der Urin war eiweisshaltig, der Puls sehr beschleunigt, bis auf 132 Schläge in der Minute. Die Untersuchung des Nervensystems ergab keinen besonderen Befund. An der rechten Stirn- 
seite war eine Wunde sichtbar. Die Haut um das rechte Auge herum war angeschwollen und blau verfärbt, die Haut oberhalb des linken Fussrückens war blaugrün, die grosse Zehe rechts blauroth verfärbt und geschwollen.

Patient gab einen täglichen Schnapsconsum von $30-40$ Pf. zu. Von früheren Krankheiten wusste er nichts zu berichten, ausser dass er einmal einen weichen Schanker gehabt hätte. Das Delirium hielt mit ausserordentlicher Intensität an. In seiner tobsüchtigen Erregung stiess Patient einmal mit dem Kopf gegen die Wand, sodass eine Schwellung des ganzen Gesiohts, besonders der Stirn und auch eine neue kleine Wunde an der Stirn entstand.

Am 15. Februar wurde zuerst eine linksseitige Lähmung bei dem Patienten bemerkt. Patient war vollständig benommen, sodass er auf Fragen nicht antwortete. Gegen tiefe Nadelstiche machte er keine Abwehrbewegungen. Die Schwellung an der Kopfhaut wurde stärker und es bildete sich hier eine Phlegmone ans, die in der chirurgischen Klinik behandelt wurde. In den nächstfolgenden Tagen bis zu seinem Tode am 8. März nahm die Benommenheit immer mehr zu. Zu der Lähmung im linken Arm und linken Bein kam noch eine Lähmung im linken Facialis hinzu.

Bei der Section wurde eine Phlegmone des Kopfes constatirt, ferner eine linksseitige fibrinöse Pleuritis, eine doppelseitige bypostatische Pneumonie, Myocarditis, Nephritis, Gastroenteritis und Milzanschwellung. Das Schädeldach war dick und schwer. Es fand sich eine geradlinige Fissur, die im rechten Schläfenbein nach links binten verlief und in der linken Parietalgegend endete. Die Fissur betraf nur die Tabula externa. Auf der rechten Seite befand sich eine Blutgeschwalst $z$ wischen der inneren Fläche der Dura mater und dem Gehirn, durchwelche der vordere Theil der rechten Hemisphärezusammengedrächt wurde.

Die Pia mater der rechten Hemisphäre ist überall stark blutig durchsetzt, und zwar hauptsächlich an den Uebergangspartien von der Convexität zur Basis. Die ganze Hemisphäre erscheint eingedrückt und missgestaltet. Man sieht zwei besonders bemerkenswerthe Einsenkungen - eine grössere in der Nähe des Schläfenlappens, eine kleinere in der Gegend der Centralwindung. Am Stirnlappen findet sich eine Höhle, welche sich schwappend anfühlt (s. Taf. XVII. Fig. 1 und 2). An der Grenze zwischen Schläfen- und Hinterhauptslappen sitzt eine Erweichung, ebenso an dem Uebergangstheil von Schläfen- und Hinterhauptslappen.

Auf Durehschnitten sieht man zunächst, dass die ganze rechte Hemisphäre zusammengedrückt ist gegenüber der linken, namentlich in den Stirnlappen erscheint die Rinde auf der rechten Seite schmäler, als auf der linken. Die Rinde ist an vielen Stellen durchsetzt mit kleinen Herden, die zum Theil eine blutige Färbung aufweisen, zum Theil gelblich und weisslich gefärbt sind. Diese Herde sind nicht nur auf der Aussenseite der Hemisphäre sichtbar; sondern treten auch an der Medianflächedes Gehirns auf.

Auch in der Basis der linken Hemisphäre sind in der Rinde einzelne kleine Herde zu bemerken. Ebenso sind Pons und Medulla oblongata von Blutpunkten durchsetzt, besonders in der Gegend der Substantia nigra. 
Was zunächst die Verbreitung der Herderkrankungen anbetrifit, so sehen wir sehr schön schon an Pal'schen Präparaten die einzelnon Herde in weisser Farbe scharf abgegrenzt innerhalb der Rinde hervortreten, und zwar mit Torliebe in den tieferen Schichten der Rinde (s. Taf. XVII., Fig. 4). Ihre Form ist unregelmässig. Die Marksubstanz ist niemals ergriffen. An einzelnen Stellen sind ganze Rindenpartien in Zusammenhang erkrankt, sodass die Rinde verschmälert erscheint und sich auf $\mathrm{Pal}$ 'schen Präparaten durch ihre helle Färbung, auf Weigert'schen Präparaten durch ihren braunen Farbenton und endlich in Säurefuchsin Präparaten durch dunkelrothe Färbung abhebt. Als dritte Art der Herdbildung finden wir endlich eine Cyste, die an der Aussenseite der rechten Hemisphäre etwa in der Parietalgegend gelegen ist.

Bei einem histologischen Detailstudium treten uns folgende eigenthümliche Processe entgegen. Erstens findet man Stellen, in denen eine blosse Ver- dichtung des Gewebes sichtbar ist, ohne dass man eine entzündliche Reaction bemerlkt. Die Stellen scheiden sich in Säurefuchsinpräparaten durch ihre dunkelrothe Färbung von don normalen Partien ab und zeigen bei stärkerer Vergrösserung eine einfache Verdichtung der Glia, die weniger maschenreich ist. Von einem erheblichen Kernreichthum kann man nicht sprechen. Eine gewisse Reaction in den Gliakernen ist insofern sichtbar, als einzelne grösser und blasiger geworden sind und blass anssehen im Vergleich mit den normalen Kernen. Ferner ist ein Gefässreichthum in den erwähnten Partien bemerkensworth; die Gefässe sind erwoitert, und es macht den Eindruck, als ob die Kerne in den Gefässscheiden erheblich vermehrt seien (s. Taf. XVII., Fig. 5 u. 6). Was das Nervengewebe anbetrifft, so ist vor allen Dingen hervorzuheben, dass die Ganglienzellen verändert sind, was sich in den Säurefuchsinpräparaten dadurch deutlich zu erkennen giebt, dass die Kerne unansehnlich, dunlielroth gefärbt sind, ein Kernkörperchen vermissen lassen, und dass die Zellkörper als scharfe eckige Figuren deutlich hervortreten. Die Nervenfasern sind zu Grunde gegangen und man findet keine Spur mehr von dem feinen Nervenfasernetz, welches die Rinde durchsetzt.

In anderen Partien ist das Bild, welches sich uns darbietet, ein etwas anderes. Die Glia hat sich in ein grobbalkiges Gerüst aufgelöst. Der Kernreichthum ist ein sehr grosser; man sieht alle möglichen Uebergänge von freien Kernen zu Kernen mit Protoplasmamasse, ganz wie man es immer sieht, wenn das Gliagowebe anfängt, gegen einen pathologischen Reiz zu reagiren. Hier und da lösen sich schon in den Maschen des Gewebes deutlich freie Zellen ab, die den Uebergang zu Rundzellen bilden, aber noch nicht den blassen, scharf contourirten Zellenleib derselben aufweisen. Die Gefässe sind in diesen Gewebspartien ausserordentlich vermehrt, zum Theil mit Blut gefüllt und von starken Gefässscheiden umgeben.

Endlich im dritten Stadium sieht man in einer stark mit Gefässnetzen durchsetzten Partie, in der die Glia nun gelatinös aussieht und die gewöhnliche Structur vollständig verloren hat, zahlreiche Rundzellen von dem bekannten Aussehen mit hellem, scharf contourirtem Zellenleib, ganz so wie man 
das bei einer gewöhnlichen Encephalitis findet (s. Taf. XVII., Fig. 7). Die veränderten Gliazellen sind in solchen Partien weniger sichtbar.

Die Wirkung des Druckes, den ein Hämatom auf das Gehirn ausübt, ist durchaus nicht gleichartig. Gewöbnlich - und ich habe selbst Gelegenheit gehabt solche Fälle zı sehen - drückt ein Hämatom einfach die Gehirnrinde zusammen, obne zunächst gröbere Veränderungen hervorzurufen.

Ich habe dann selbst Veränderungen beschrieben, die in dem Gehirn eines Neugeborenen zu beobachten und auf den Druck eines Hämatoms zurückzuführen waren ${ }^{1}$ ). Die vorstehende Untersuchung stellt einen sehr ähnlichen Befund im ausgewachsenen Gehirn fest. Wäre unter günstigen Umständen eine Heilung des Processes zu Stande gelsommen, so hätte sich eine Sklerose entwickeln müssen, durch die ganze Rindenpartien in Atrophie verfallen wären, also ein Befund, der öfters erhoben ist, ohne dass man bisher eine sichere Vorstellung über die Entstehung solcher Processe gewinnen konnte. Wir selbst haben in einem solchen Fall ron Sklerose bereits auf die Möglichkeit dieser Fntstehung hingewiesen.2)

Was num die Art des hier vorliegenden Processes anbetrifft, so sehen wir alle mögliche Formen der Rindenveränderungen. An einzelnen Stellen ist die Glia einfach verdichtet, ihre feine Spinngewebsstructur ist verloren gegangen und die Nervenelemente sind abgestorben aus Mangel an den nöthigen Lebensbedingungen. Die Ganglienzellen zeigen einfache Sclerose oder Schrumpfung. Die Lage solcher Herde legt oft die Vermuthung nahe, dass ein corticales Gefäss durch den Druck der Neubildung versperrt worden ist and dass das von ihm versorgte Gebiet verödete. Die sonstigen Veränderungen, die sich in der Rinde finden liessen, stellen Uebergangsstadien dar von einem einfachen Absterben des Gewebes bis zur lebhaftesten Reaction des Gewebes gegen den gesetzten Reiz, so dass schliesslich auch stellenweise eine regelrechte Eneephalitis zur Entwickelung gekommen ist. Würden sich blos derartige encephalitische Veränderungen der Gehirnsubstanz gefunden baben, so liesse sich ja immer die Vermuthung aufstellen, dass nicht der einfache Druck die Rindenveränderung verursacht habe, sondern dass in diesem Falle eine infectiöse Miterkrankung der Gehirnrinde vorliege, da wir ja bören, dass Pat. an einer phlegmonösen Erkrankung der Kopf haut litt. Allein diese Annahme ist gänzlich auszuschliessen, einmal wegen des Fehlens

1) Dieses Archiv. 1898.

2) Dieses Archiv Bd. 28. 
einer eitrigen Meningitis und dann spricht dagegen der Befund gänzlich reizloser Veränderungen an einzelnen Stellen der Gehirnrinde.

Das Endproduct aller dieser Processe ist aber in letzter Linie immer eine Sklerose und dadurch bedingte Verkümmerung der Hirnrinde. Die Entstehung durch den Druck eines Hämatoms muss jedenfalls immer in Betracht gezogen werden, falls es sich um die Erklärung von ausgebreiteten alten Rindenatrophien handelt, namentlich dann, wenn die mikroskopische Untersuchung scharf ausgeprägte sclerotische Herde in der Hirnrinde erkennen lässt.

\section{Erklärung der Abbildungen (Taf. XVII.).}

Figur 1. Aussehen der durch das Hämatom zusammengedrückten rechten Hemisphäre. Vorn eine Cyste.

Figur 2. Querdurchschnitt durch das Gehirn von vorn geschen. Rechte Hemisphäre im Ganzen zusammengedrückt. Cyste in der Hirnrinde. Die dunkelen Stellen in der Hirmrinde sind Herdbildungen, die Rinde zum Theil ausserordentlich verschmälert. Die ausgedehnten dunkelen Partien $\mathrm{H}$ zeigen hier im Wesentlichen eine Veränderung des Gewebes wie sie in Figur 6 abgebildet ist.

Figur 3. Querdurchschnitte zeigt ebenfalls die Ausbreitung der Herde in der Rinde.

Figur 4. Schnitt nach Pal gefärbt. Die weissen, wie ausradirt aussehenden Stellen Herdbildungen.

Figur 5. Veränderte Hirnrinde in der Tiefe einer Furche bei schwacher Vergrösserung. Die pathologischen Stellen sind durch eine scheinbare, oder wirkliche Vermehrung der Gliakerne ausgezeichnet.

Figur 6. Aus einer veränderten Rindenstelle. Hier im Wesentlichen Verdichtung der Glia, Zusammendrängen der Kerne und Hervortreten von weiten Gefässen. a sklerosirte, $b$ normale Gliasubstanz.

Figur 7. Aus einer veränderten Stelle der Gehirnrinde. Hier hat der Process den Charakter der Encephalitis. Gliakerne mit Protoplasma und freie Rundzellen. 


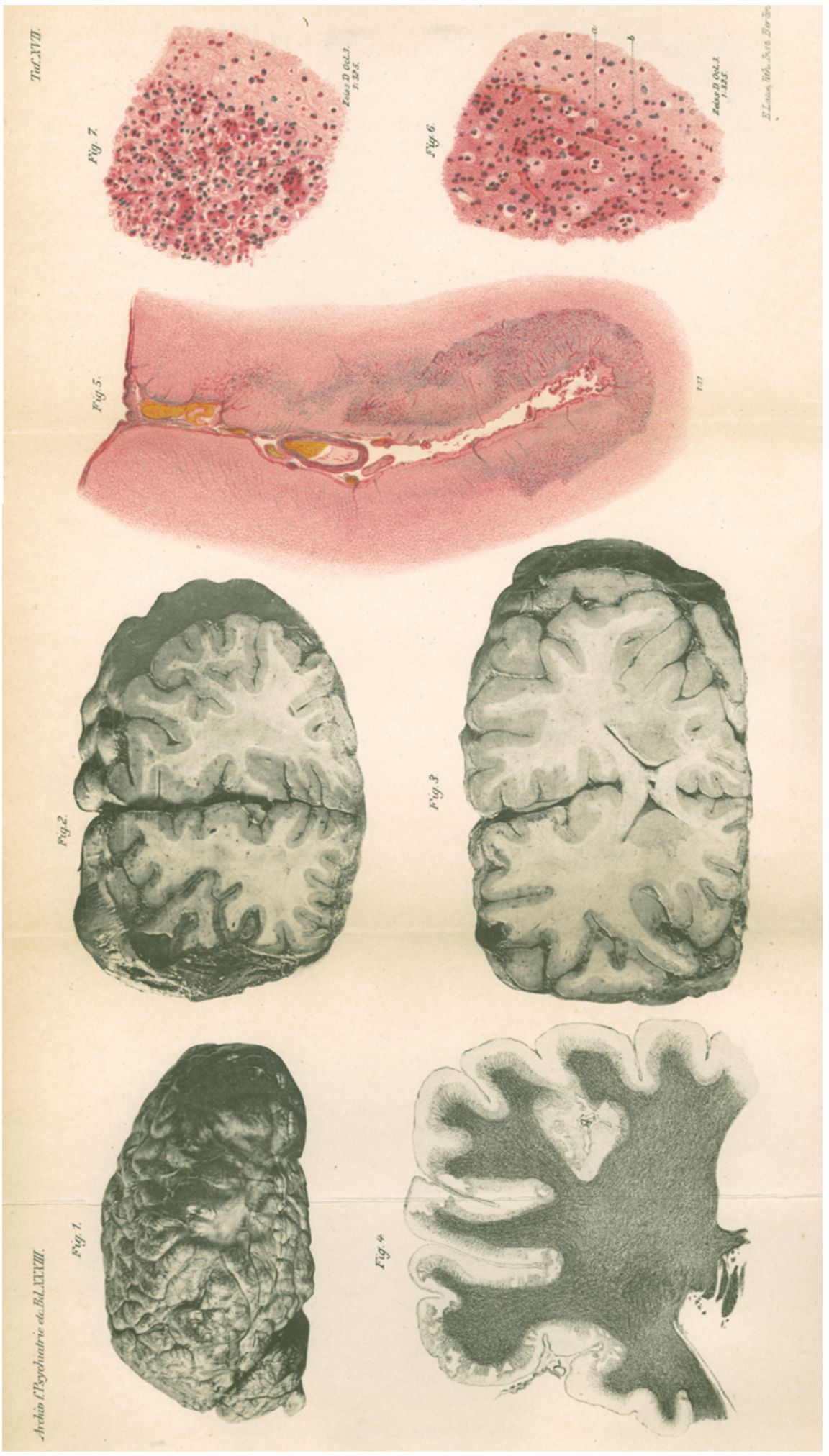

